\title{
Directions of cosmic anisotropies
}

\author{
Katarzyna Bajan ${ }^{1}$, Piotr Flin ${ }^{2,3}$ \& Victor N. Pervushin ${ }^{2}$ \\ 1. H. Niewodniczanski Institute of Nuclear Physics, ul.Radzikowskiego \\ 152, Krakow, 31-342, Poland \\ 2. Bogoliubov Laboratory of Theoretical Physics, Joint Institute for \\ Nuclear Research, Dubna, Moscow Region 141980, Russia \\ 3. Pedagogical University, Institute of Physics, ul.Swietokrzyska 15, \\ Kielce, 25-406, Poland
}

\begin{abstract}
We argue that the recently reported anisotropy of the local velocity field is in good agreement with other cosmic anisotropies and they are all explainable by the Great Attractor.
\end{abstract}

The position of the Great Attractor (Lynden-Bell et al. 1988), i.e. of a huge mass concentration, causing the large-scale streaming motion observed in the Local, Perseus-Pisces and Hydra-Centaurus superclusters, is as expressed in the supergalactic coordinate (Faber et al. 1988) $L=161^{\circ}, B=-6^{\circ}$. Another important direction in the sky is that of the dipole moment of the cosmic microwave background radiation. It is generally interpreted as a Doppler shift resulting from the motion of the Sun with respect to the co-moving reference frame. The direction of the dipole anisotropy (Lineweawer 1996) can be written as $L=117^{\circ}, B=-27^{\circ}$. In these coordinates the position of the Virgo cluster is: $L=103^{\circ}, B=-2^{\circ}$.

Recently, anisotropy of the local velocity field was detected (Karachentsev 2001) studying the neighbouring region of the Universe, with galaxies of radial velocities $V_{r}<500 \mathrm{~km} \mathrm{~s}^{-1}$. The photometric distances to galaxies were determined using various methods. Moreover, their radial velocities were also determined. This allows one to show clear anisotropy in the value of the Hubble constant, which corresponds to the anisotropy of the local velocity field. This peculiar velocity field can be described by a tensor of the local Hubble parameter with the mutually perpendicular principal axes of values: 81,62 and $48 \mathrm{~km} \mathrm{~s}^{-1} \mathrm{Mpc}^{-1}$ with a standard error of $\sim 4 \mathrm{~km} \mathrm{~s}^{-1} \mathrm{Mpc}^{-1}$. It was noted (Karachentsev 2001) that the major axis of the ellipsoid is directed only $29^{\circ}$ away from the Virgo cluster centre, while its minor axis is directed almost along the polar axis of the Local Supercluster. Departure from the ideal, uniform and isotropic, Hubble flow was noted previously (de Vaucouleurs 1958, 1985) and it was suggested (de Vaucouleurs 1985) that it can be connected with the peculiar motion of the Local Group of galaxies relative to the Local Supercluster objects, due to mass concentration (Tully 1988).

In the present paper we point out several interesting coincidences connected with possible mass concentrations. The coordinates of the local anisotropy determined by Karachentsev are $L=131^{\circ}, B=0^{\circ}$. The position of the three apices on the celestial sphere and the centre of the Virgo cluster (which is the 


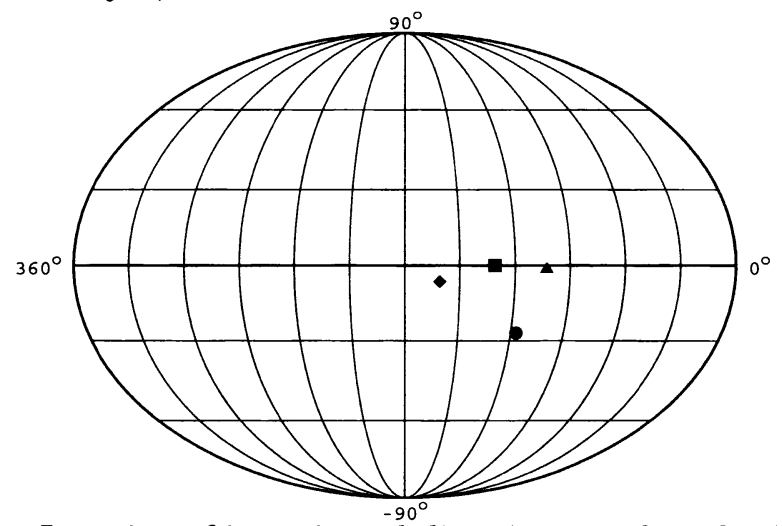

Figure 1. Location of investigated directions on the celestial sphere. The triangle denotes the position of the Virgo Cluster; circle - MBR direction; square - the Karachentsev's anisotropy; rhombus - the Great Attractor.

centre of the Local Supercluster), are close together as well as they are close to the main plane ("equator") of the Local Supercluster.

We think that the coincidence of directions connected with the radial velocities of galaxies is not unexpected (Scaramella et al. 1989). It is due to the great mass concentration called the Great Attractor, which influences all objects, causing a large-scale streaming motion of galaxies, observed in the linear scale almost two orders of magnitude greater than that detected by Karachentsev. The difficulties in observational locating the Great Attractor can be connected with the fact that the Great Attractor does not consist of luminous, but of dark matter. This suggestion is strongly supported by the contribution of baryons to the total energy density of the Universe. It is commonly accepted (Lahav 2002) that $\Omega_{b} h^{2}=0.02$, which for $h=0.75$ gives $\Omega_{b}=0.03$ and 0.05 for presently favourable $h=0.65$.

\section{References}

de Vaucouleurs, G. 1958, AJ, 63, 253

de Vaucouleurs, G., Peters, W. L. 1985, ApJ, 297, 27

Faber, S. M., Burstein, D. 1988 in Large Scale Motions in the Universe, eds. G. Coyne \& V. Rubin, Princeton Univ. Press, 116

Karachentsev, I. D. 2001, Usph. Phys. Nauk, 171, 860

Karachentsev, I. D., Makarov, D. I. 2001, Astrofisica, 44, 5

Lahav, O. 2002, XXXVII-th Recontres de Moriond, The Cosmological Model, astro-ph/0208297

Lineweawer, C. H., et al. 1996, ApJ, 470, 38

Lynden-Bell, D., et al. 1988, ApJ, 326, 19

Scaramella, R., Baiesi-Pillastrini, G., Chincarini, G., Vettolani, G., Zamorani, G. 1989, Nature, 338, 526

Tully, R. B. 1988, Nature, 334, 209 